\title{
Sachregister zu Band 59
}

Die fett gedruckten Zahlen bezeichnen Eigenarbeiten. Bb. = Buchbesprechung.

A.

Aalblutserum, Einfluß des - auf

das Auge 81. Ablatio retinae go, 91, 194, 402. Aceton, Hornhauterosionen als Fol-

ge von Anwendung von - 205. Adaptationszustand, Abhängig-

keit der Sehschärfe bei Medien-trübungen vom - 344. Aderhaut s. Chorioidea. Akinesie 207.

Akkommodationslähmung, ein-

seitige - nach Diphtherie 379. Aktinomykose in Polypen des obe-

ren Tränenröhrchens 376. Albinismus, operative optische

Therapie des totalen - 388. Aluminium, Verhalten des - im

Auge 310. Ammoniakverletzung, beider-

seitige Katarakt durch - 311. Argyrose, hämatogene - der Hornhaut 188. Arteria centralis

retinae, Em-

bolie der $-89,90$.

Embolie der - mit gleichzeitiger Verstopfung hinterer Ziliar-arterien 320.

Messung des Blutdrucks in der - 107.

Arterien, Spasmus der retinalen -

I3i.

Atheromatose der Hornhaut mit

Cholesterinkristallen in der Vor-

derkammer 86. Atropinbehandlung bei Irido-

cyclitis glaucomatosa 129, 130. Atropin-Glaukom, Irisatrophie

nach - 189. Augapfel s. Bulbus. Augenbewegungen, ungewöhn-

liche Phänomene der 197.

- $\quad$ Untersuchung der - 112.Augenlider s. Lider.Augenspiegel, Verbesserung des

elektrischen - 116. Augenverletzungen 101, 102. Automobilunfall des Auges 311. Axonoskop 115 .

B.

Bakterien, Lichtwirkung auf-

374-Bandkeratitis 75. Bazillenemulsion 385. Bindehaut s. Conjunctiva. Binokularsehen 206.

- $\quad$ Untersuchung des -. 112.Blastome der Orbita 98.Blaue Sklera und Knochenbrüchig-

keit 181, 323.

Blausäurevergiftung durch ein Schuhputzmittel 335.

Blennorrhoea neonatorum bei einem Kaiserschnittskind 211.

Blepharochalasis 328.

Blepharoconj unctivitis angular is, Wirkung von Zinksulfat auf die -210 .

Blepharospasmus, essentieller

315-

Blickfeld, das praktische -113 , 
114. Blink-Farbenlaterne 116. Blutbewegung im Auge, entop-

tische Sichtbarmachung der -

380. Blutdruck in der Arteria centralis

retinae 107. Blutimbibition der vorderen Lin-

senkapsel 85. Blutung, retrochorioideale - unter

dem Bilde eines Chorioidealtu-

mors 401. Botulismus, Augenveränderungen

bei -103 , 308. Brillenbestimmung 115, 116. BulbuseröffnendeÕperationen,

bakteriologische Prophylaxe vor

-39 a. Bulbuszyste 103.

C. Chiasma, Schema des Verlaufs der

Nervenfasern durch das -185 . Chlorkalzium, Verbrennung der

Augen durch - 311. Cholesterinausstreuung im

Kammerwasser bei hypermaturer

Katarakt als Ursache von Sekun-

därglaukom 397. Cholesterinkristalle in der Vor-

derkammer 86. Chorioidea, eigenartige Streifen-

bildung in den äußeren Schichten

der -322 .

- $\quad$ Flächensarkom der -121.

Sarkom der - 81, 83, 195, 201.

Tumor der - $\square$ vorgetäuscht durcheine retrochorioideale Blutung 401 .

Chorioiditis, Dif $f$ erentialdiagnose zwischen Drüsen der Aderhaut und - 60 .

Conjunctiva, chronische ge-

schwulstartige Tuberkulose der -

79-

Initialsklerose an der -376 .

Pathologie der - $176 \mathrm{ft} ., 371,372$.

pathologische Anatomie der $-69 \mathrm{ff}$.

Tuberkulose der - 71, 79, 176, 373-

$27 *$

406

Sachregister zu Band 59.

Conjunctivallappen, Zystenbil-dung in einem Kuhntschen - 202.

Conjunctivitis, chronische mem-branöse 375 .

- $\quad$ follicularis und Trachom 366.

- metastatische - bei akutem Ge-lenkrheumatismus 178, 374.

- $\quad$ Schwimmbad-C. 126, 367.Cornea, Erosionen der - als Folge

von Acetonanwendung 205.

-abszeß, akuter, metastatischer syphilitischer -. 188, 376.

-geschwüre, Erfahrungen mit der Sondermannschen Trepanation bei a- 35 .

-krümmung, eine dem Kerato-konus entgegengesetzte - 186.

Pathologie der - 184Íf.

Pathologie der -372.

pathologische Anatomie der - 74 if. 
Pigmentbelag an der Hinterwand der - 397.

rezidivierende Iridocyclitis durch einen Glassplitter in der -314 .

Transplantation nach Verätzung

387 .

- $\quad$ Verbrennung der - - durch künst-liche Höhensonne 308.

- $\quad$ Verkupferung der-77,310,311.Corpus callosum, Sarkom des -

100. Corpus ciliare, Mißbildung des - in einem Mikrophthalmus 81.

- $\quad$ traumatische Nekrose des - 80.Corpus vitreum - Absaugung

oder Sklerotomie 393. -- lebende Fliegenlarve im - 376.

- $\quad$ Pathologie des a- 192, 193.Curral 384.

D.

Dakryozystorhinostomie 395, 396.

Degeneratio disciformis maculae luteae 337 (Bb.).

Dermoidzyste, ein Zahn in einer epibulbären - 77, 200.

Diphtherie, einseitige Akkommo-dationslähmung nach - 379 .

- $\quad$ und Gonoblennorrhoe der Binde-haut 372.

Diplobazillen-Protease 210. Diszission 393. Doppeltsehen, monokuläres 182.

- postoperatives 122 .

- $\quad$ Prüfung auf - 113.Drehrad, optisches - mit elek-

trischem Antrieb 324.

Druck, intraokularer - bei retro-bulbären Aífektionen 210.

Drusen der Aderhaut, Differential-diagnose zwischen zentraler Cho-rioiditis und - 60 .

- $\quad$ der Papille 206.Dystrophia corneae 75.Dystrophie, myotonische - mit

Katarakt 125.

E. Echinococcus, intraokularer 200,

375-Elephantiasis, einseitige -- des

Oberlides bei Erweiterung der

Sella turcica 305. Elliotoperation, Spätinfektion

nach - 375. Embolie der Árteria centralis retinae 89, 90, 339. Encephalitis lethargica, Augen-

komplikationen als Spätfolgen

von $-312,378$.

- $\quad$ schnell reifende Katarakt bei -191 .

Encephalocele orbitae posterior 121.

Entropium, operierter Fall von - 202.

Epinephrin zur Behandlung der Iridocyclitis 130.

Episkleritis metastatica 374,

378 Epitarsus, der sogenannte - 50. Epithelfasersystem der Horn-

haut 74. Exophthalmus, einseitiger trau-

matischt $\cdot r-304$.

- $\quad$ Erblindung infolge doppelseiti-gen - hervorgerufen durch einenPseudotumor orbitae 98.

$\Gamma$.

Farbenlehre Goethes 124. Färbung, vitale 117. Fixierzügelnähte bei Lidopera-

tionen 386. Fliegenlarve, lebende - im Glas-

körper 376. Folliculosis conjunctivae 316.

G.

Gelenkrheumatismus, metastatische Conjunctivitis bei akutem 


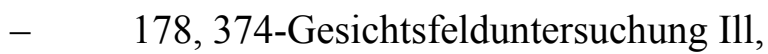

112. Glaukom 100, 101.

bei "Verkupferung des Auges 310..

Beziehung der Hypophysis zum

- 306.

- Cholesterinausstreuung im Kam-merwasser bei hypermaturer Katarakt als Ursache des

Sekundär-

- 397-

Heterochromie-G. 399.

Holths tangentiale Sklerektomie bei -128 .

Glaukosan 122.

Gliom der Iriswurzel 81, 189.

Glioma retinae bei einem sieben-

jährigen Kinde 125. Goethes Farbenlehre 124. Goldchloridfärbung 203, 325,402.

- $\quad$ eines Leukoma adhaerens 119.Goldsolreaktion im Liquor 382. Gonoblennorrhoe bei

Kaiser-

schnittskind 211.

Sachregister zu Band 59.

407

Gonoblennorrhoe, Olobinthin bei - 319 .

- $\quad$ und Diphtherie der Bindehaut372-

GranulationsgeschwulstinOber-lid und Orbita nach Verletzung mit korpuskulärem Farbstoff

(Tin-tenstift) 64.

$\mathrm{H}$.

Ha are, Resorption der - in der

Hornhaut 184. Hämangio-Endothelioma peri-

vasculare der Orbita 98, 303. Hautverbrennung, Neuritis retro-

bulbaris bei - 196. Hedonal, rektale Einschläferung

mit - 382. Hemianopsia inferior, ein Fall

von ungewöhnlicher - 145 . Hemihypertrophie der oberen

Körperhälfte infolge von Geburts-

trauma 312. Herpes corneae, latenter Aufent-

halt des Herpesvirus im Conjunc-

tivalsack bei posttraumatischem

- 374-

- Pathogenese des hämatogenen -

84. 307, 364- Herpetiforme Erkrankung der

Hornhautrückfläche 185. Heterochromieglaukom 399. Hippelsche Krankheit 124. Hirntumor, Zentralskotom bei -

196. Höhenschielen, scheinbares 199. Höhensonne, Verbrennung der Cornea durch künstliche 308. Hornerscher Symptomenkom-

plex bei Hemihypertrophie der

oberen Körperhälfte 312. Hornhaut s. Cornea. Hornhautring, Kayser-Fleischer-

scher - bei Pseudosklerose 76. Hyaline Bildungen, Entstehung

der - im Auge 89. Hyalitis bei Meningitis cerebro- 
spinalis epidemica 90. Hyperphorie, doppelseitige kon-

genitale (familiäre) Trochlearis-

lähmung und ihre Beziehung zur

alternierenden - 17. Hypoderma bovis, lebende Larve

von - im Glaskörper 376. Hypophysis und Auge 306. Hypophysistumoren 99, 100, 305.

- Bestrahlung der - 327.Hypotonia acuta, tonische Iritis

nach Netzhautablösung bei -

i95.

I.

Ichthyosis der Hornhaut 185.

- und Bindehauttuberkulose 373.Implantationszysten, subkon-

junktivale - 72, 178. Indigoblaufärbung des Auges

3.99-Infektionsübertragung, experi-

mentelle - von Bulbus zu Bulbus

84,36 .

Initialsklerose an der Bindehaut

.376. Iridektomia limbaris 390 Iridocyclitis, rezidivierende - - durch einen Glassplitter in der

Hornhaut 314

- $\quad$ unreife Katarakt als Ursache von-203.

Iridocyclitis glaucomatosa, Atropinbehandlung bei - 129. 130.

- $\quad$ Behandlung der -- 190.Iridodialyse mit Sphinkterriß 156.Iris, angeborene Hypoplasie des vorderen Blattes der - mit My-driasis 201. - Melanose und Sarkose der - 82.

- mikroskopische Anatomie der-

212 (Bb.). - - Pathologie der $-189,190 .-\cdot$ traumatische Nekrose der - . 80. Irisatrophie bei

Glaukom 100.

-genuine 202. Irisgefäße, ziliare - - als kongeni-

tale Anomalie 79. Irisschnitt, limbarer - 390. Iritis, toxische - nach Netzhautablösung bei

Hypotonia acuta

Kanthoplastik mit Bildung einer

Caruncula 202. Kapillarmikroskopische Be-

funde 379. Katarakt 191.

bei Strumektomie 357.

Cholesterinausstreuung im Kam-merwasser bei hypermaturer - als Ursache von Sekundärglau-

kom 397.

mit myotonischer Dystrophie 125.

unreife - als Ursache von Irido-cyclitiden 203.

Kataraktoperationen, Nebén-verletzungen bei - 102.

- $\quad$ Spätinfektionen nach normal ver-laufener - 375.

- $\quad$ Verbesserung der - 391, 392.Keratektasie nach Randatrophie

182. Keratitis dendritica 182. Keratitis filiformis 184.

interstitialis punctata bei Lues 188 .

parenchymatosa nodularis bei Lues congenita tarda 76 .

Keratoplastik 388. Knochenbrüchigkeit, Otosklero-

se und blaue Sklera 181, 323 Koch-Weekssche Bazillen 372,

376-

Konvergenzkrampf 400. 
Kosmetische Operationen 326.

Kupferammoniumsulfat, Behandlung des Trachoms mit intra· venösem -ロ 161.

L.

Lenticonus posterior 20

408

Sachregister

zu Band 59.

Leukomaadhaerens, Goldchlorid-

tätowierung eines - 119. Lichtwirkung auf Bakterien 374. Lider, Dunkelfärbung beider- 175.

- phlegmonöse Entzündung der -328.

Lidkarzinom, Behandlung des -

329-Lidoperationen, Fixierzügelnähte

bei - 386. Lidschließer und Lidspalte 1. Linse, Wirkung der Serumtoxine

auf die - 85. Linsenektopie, Spaltlampenbe-

fund der Zonula Zinnii bei an-

geborener - 201. Linsenepithel, traumatische Ne-

krose des -80 . Linsenkapsel, Blutimbibition der

vorderen -85 . Linsentrübung, Pathologie der

angeborenen -85 . Lues, einige seltene luet. Erkran-

kungen des Auges 213. Luxatio bulbi 329. Lymphozytom, epibulbäres - 77.

M.

Maculalutea, Anatomie der -333 .

Bindegewebe vor der -322 .

Degeneratio disciformis der - 337 (Bb.).

tapetoretinale Degeneration der- 320 .

Maculaarterie, Embolie einer - ohne Gefäßveränderung 339.

Makulakolobom 315.

Magnetoperation in.

Medialisparese, einseitige supra-nukleare - 197.

Megalocornea 202.

Melanosarkom am Sehnervenein-tritt 83.

- $\quad$ der Iris 82, 189.Melanose der Iris 82.Membrana Deszemeti, Glaslei-

stenbildung der - infolge von

Geburtstrauma 312. Risse und Abhebungen der-

183, 186. Meningitis tuberculosa, Beteili-

gung des Optikus bei der - 94. Mikrophthalmus, Mißbildung der

Retina und des Ziliarkörpers in

einem - 81. Milchinj ektionen 383. Mißbildung des Ziliarkörpers und

der Retina im Innern eines

Mikrophthalmus 81. Mitbewegungen des Oberlides 175. Multiple Sklerose, Bedeutung der Dauer eines zentralen Skotoms

bei der Neuritis retrobulbaris für

die Diagnose der - 313. Musculusobliquus inferior, My-

ektomie des -127 . Raddrehung des Auges bei

Funktionsstörung des -205 . 
Musculus rectus externus, Strangfixation des -127 .

Myektomie des M. obliquus inferior 127.

Myotonische Dystrophie mit Katarakt 125.

N. Naevus der Conjunctiva bulbi 72.

- $\quad$ rasches Wachstum eines Limbus-sarkoms im Anschluß an einen -

376 - Nasennebenhöhlen, Pathologie

der - bei der rhinogenen retro-

bulbären Neuritis 100. Nebennieren, abnormes Längen-

wachstum der Wimpern bei Über-

funktion der - 354. Nekrose, traumatische - der Uvea

und des Linsenepithels 80. Nervenfasern, Schema des $\mathrm{V}^{7} / 8 \mathrm{r}-$

laufs der - durch das Chiasma

135 .

- $\quad$ ungewöhnliche Heteropie mark-haltiger - 88 .

Nervus oculomotorius, Kern-gebiet des - 96.

- flüchtige einseitige Lähmung des-399-

Nervus opticus, endonasale Ein-griffe bei Erkrankungen des -

394-ロ- traumatisches Hämatom der

Scheide des - 92. -- Stammbaum bei Leberscher he-

reditärer -125 .

- tabische 94.

-atrophie mit Arteriosklerose bei Diabetes 94.

Netzhaut s. Retina.

ablösung s. Ablatio retinae.

Neuritis retrobulbaris, Bedeutung der Dauer eines zentralen Skotoms bei der - für die Diagnose der multiplen Sklerose 312.

bei Hautverbrennung 196.

Pathologie der Nasennebenhöhlen bei der rhinogenen - 100 .

Neurodermitis, Katarakt bei- 191. Nystagmus als Gehirnstrahlung 132 (Bb.).

- $\quad$ alternans 199.

0.

Oberlidrandlinie 182. Oguchische Krankheit, Sek-

tionsfall von - 89. Olobinthin bei Gonoblennorrhoe

3i9. Operationen, bakteriologische Pro-

phylaxe vor bulbuseröffnenden --

390. Ophthalmia sympathica 84,307 ,

$332,3^{\circ} 4 \cdot$ Ophthalmoplegie bei Lues cere-

bri 125. Ophthalmoskopie im rotfreien

Licht 106.

Sachregister zu Band 59.

409

Opticus s. Nervus opticus. Orbitalphlegmone 328. Orbitaltumoren 98, 99, ioo, 124.

Operation der -394.

Röntgenbehandlung bei - 384. Otosklerose und blaue Sklera 181.

P. 
Panophthalmitis tuberculosa

8o. 373-Paralyse, die periphere Sehbahn

bei - 95. Parasitologie des Auges 364ft. Parinaudsche Conjunctivitis

205. Parotitis mit Uveitis 125. Partialantigene von Deycke zur

innerlichen Behandlung der Au-

gentuberkulose 290. Pech, Augenerkrankungen durch -.

$3^{\circ} 9 \cdot$ Pemphigus der Conjunctiva 71. Perimeter Ill.

Perivaskuläre Räume, im Axial-strange, Verbindung der - mit

dem Zwischenscheidenraum des

Optikus 92. Phlegmonöse Entzündung der

Lider und der Orbita 328. Photodynamische Wirkung auf

Bakterien 374. Photographie des lebenden Auges

no. Pigmentbelag an der Hornhaut-

hinterwand 397. Pigmentverschiebung im Reti-

nalepithel 89. Pilzerkrankungen des Auges 376. Plica semilunaris. eine Anomalie

der - 50. Pneumokokken-Blennorrhoe

der Neugeborenen 177 369. Ponndorfbehandlung 208. Preglsche Lösung zur Behandlung

eitriger Tränensackleiden 176. Prismenoptometer 114. Proteinkörpertherapie 383.

Pseudopterygien, Beseitigung der

- durch Thiersch sche Läppchen

387. Pseudosklerose mit Kayser-

Fleischerschem Hornhautring 76. Pseudotumor orbitae 91.

entziindlicher -331 .

Erblindung infolge doppelseitigen Exophthalmus, hervorgerufen durch einen - 98 .

Psychophysik augenärztlicher Un-tersuchungsmethoden, 117.

Pterygium, Entwicklung und Behandlung des -285.

Ptosis auf myasthenischer Grund-lage 387.

- $\quad$ operierter Fall von -- 202.Pupillen, geschlitzte 125.Pupillenreaktion, paradoxe 197.

Q.

Quecksilber im enukleierten Auge 124.

ß.

Raddrehung des Auges bei Funk-tionsstörung des M. obliquus superior 205.

Radiumbestrahlung bei tuber-kulösen Augenerkrankungen 313.

Radiumschädigung des Auges 308

Retina, Mißbildung der - in einem Mikrophthalmus 81.

- $\quad$ pathologische Anatomie der $-87 \mathrm{ff}$.

- $\quad$ Spasmus der Arterien der-131.Retinalgefäße, Verschluß der -

208. Retinitis albuminurica 126. Retino-Chorioiditis juxtapa-

pillaris 195. Retrobulbäre Affektionen,

intraokularer Druck bei - 210. Retraktionsbewegungen 197. Retrochorioideale Blutung unt.

dem Bilde eines Chorioidealtu-

mors 401. Riesenzellenin der Vorderkammer

eines wegen Hornhautgeschwürs

enukleierten Auges 86. Röntgenaufnahmen, stereoskopi-

sche - am Schädel 204. Röntgenbehandlung 384. Röntgenschädigung des Auges

204, 308. Röntgenuntersuchung no. Rumpfkompression, Stauungs- 
blutungen nach -336 .

S. Sadebaumvergiftung, Erblindung nach - 309 Salvarsanschädigungen 119. Sanokrysin bei Augentuberkulose

334-Sarkom der Chorioidea 81, 83, 195, 201.

extraokuläres 97, 199.

Flächen- - der Chorioidea 121.

- $\quad$ intraokulares - mit konseku-tiver Uveitis 83.

Sarkose der Iris 82. Scheibenartige Entartung der

Netzhautmitte 337 (Bb.). Schielen, vestibuläres 325. Schieloperationen, Dauererfolge

der-386. Schwimmbad-Conjunctivitis

126, 367. Scintillatio corporis vitrei 86. Sehbahn, die periphere - bei

Tabes und Paralyse 95. Sehleitung, anatomische Darstel-

lung des kortikalen Abschnittes

der - 95. Sehschärfe, Abhängigkeit der -

bei Medientrübungen vom Adap-

tationszustande 344 .

- $\quad$ Bestimmung der - 115.Sehstörung, eineeigenartige - 330.Sella turcica, Erweiterung der mit einseitiger Elephantiasis des Oberlides 305.

410

Sachregister zu Band 59.

Selbstverstümmelung 326. Serumtoxine, Wirkung der - auf

die Linse 85. Siderosis bulbi 102. Signalgläser 205. Silberniederschlag auf der Horn-

haut 124. Sinus frontalis, osteoplastische

Operation bei traumatischer Er-

krankung des - 394. Skier a, blaue - im Zusammenhang

mit Knochenbrüchigkeit und Oto-

sklerose 181, 323. Sklerektomie, Holths tangentiale

- $\quad$ bei Glaukom 128.Skleritis posterior 181.Sklerokeratitis tuberkulosa,

durch ein Trauma verursacht 336.

- $\quad$ pathologische Anatomie der - 76.Sklerokorneotomie bei Glaukom

101. Sklerotomieoder Glaskörperabsau-

gung 393. Skotom, Bedeutung der Dauer ernes

zentralen - bei Neuritis retro-

bulbaris für- die Diagnose der

multiplen Sklerose 312.

- $\quad$ zentrales - bei Hirntumor 196.Spaltlampe 108, 109, 126, 184.Spätinfektionen nach Operatio-

nen 375. Sphinkterriß bei Iridodialyse 156. Staphylokokkensepsis, Metasta-

sen bei - 374. Staphylom, Operation des - 387. Stauungsblutungen nach Rumpf-

kompression 336. Stauungspapille bei echtem

Zwergwuchs 121. Stereoskopische Aufnahmen des

Auges 182. Strabismus s. Schielen. Strangfixation des M. rectus ex-

ternus 127. Streptokokkensepsis, Metasta-

sen bei -. 374. Strumektomie, Katarakt bei -35 7. Sympathisierung, expe $\pi$ mentelle

- des Kaninchenauges 83. 
$\mathrm{T}$.

Tabes, die periphere Sehbahn bei

- $\quad$ 95Tarsustuberkulose 71, 373.Tätowierung, eine neue Methode

der-388, 389. - - mit Goldchlorid 119, 203, 325, 402. Teer, Augenerkrankungen durch 309 .

Tenonitis 210 .

Tintenstift, Granulationsge-

schwulst in Oberlid und Orbita nach Verletzung mit - 64.

Tonometer 106, 107.

Totische Operation 395, 396

Trachom 204, 366.

- Behandlung des - mit intra-venösem Kupíerammoniumsulfat161.

Trachom, Hornhautveränderungen

bei - 184. -- in Oberschlesien 117. - - pathologische Untersuchungen

bei -71 . Tränendrüse, Histologie der -73 .

- $\quad$ Tuberkulose der -73 .

- Überfunktion der - bei Kera-titis filiformis 184.

Tränenleiden, operative Behandlung der -396.

Tränenpunkte, Abweichungen im Stande der - 207.

Tränenröhrchen, Aktinomykose in Polypen des oberen - 376.

- $\quad$ Blennorrhoe im - 177.Tränensackfisteln, Behandlung

chronischer -300 .

Tränensackleiden, Behandlung eitriger mit Preglscher Lösung 176.

Trepanation, Erfahrungen mit der Sondermannschen - bei Ulcus serpens und anderen progressiven Hornhautgeschwüren 35.

Trochlearislähmung, doppelsei-tige kongenitale (familiäre) - und ihre Beziehung zur

alternierenden Hyperphorie 17.

Tuberkulose des Auges 373,381.

chronische geschwulstartige - der Conjunctiva 79.

der Conjunctiva 71, 176, 373.

der Netzhaut 126.

der Tränendrüse 73.

endogene - der Tarsi 71.

innerliche Behandlung der - mit Partialantigenen von Deycke 290.

intraokulare - 92, 93, 94.

Sanokrysin bei -334 .

- $\quad$ Strahlenbehandlung der - 313.Tumoren, die bösartigen - des

Auges 133 (Bb.). Tutokain 383.

U.

Ulcus serpens, Erfahrungen mit der Sondermannschen Trepanation bei - 35 .

- $\quad$ fulminans 77, 372.Unfallkunde 335.

Unf allmedizin, Handbuch der 134

(Bb.). Untersuchungsmethoden 104 Íf. Uveitis bei Parotitis 125.

$\mathrm{V}$.

Verkupferung des Auges 77.

- $\quad$ Glaukom bei - 310, 311.Versicherungskunde 335.Verweilsonde, vier Jahre lang im 
Tränenröhrchen 176.

Vitale Färbung 117.

Vitrit 205.

W.

Wespenstich, Verletzung des Auges durch 102.

Wimpern, abnormes Längenwachs-tum der - bei Überfunktion der Nebennieren 354.

Sachregister zu Band 59.

4II

Xanthoma retrobulbare 97, 200,

$3^{\circ} 3 \cdot$ Xanthomatosis bulbi in einem nicht erblindeten Auge 398.

Z. Zahn in einer epibulbären Dermoid-

zyste 77, 200. Ziliararterien, Verstopfung der -

bei Embolie der Arteria centralis

320. Zinkiontophorese bei Keratitis

dendritica 182.

Zinksulfat, Wirkung des - auf die

Blepharoconj unctivitis angularis

210. Zonula Zinnii, Spaltlampenbefund

der - bei angeborener Linsen-

ektopie 201. Zügelnähte, Abänderung der van

der Hoevenschen - 391. Zwergwuchs, Stauungspapille bei

echtem - 121. Zwillinge, eineiige 264. Zylindrome der Orbita 99. Zyste im Gebiete der oberen

Über-

gangsfalte 316. Zystizerkus, intraokulärer 375

Namenregister zu Banð 59.

Die fettgedruckten Zahlen bedeuten EiA. I Davids 394.

Abe 210. Derkac 79, 375.

Abelsdorff 94.j Deutschmann 402

Albrich 73, 376. ji.

Arnold 98, 99, 303, 305. j Egtermeyer 109.Arruga 108. 1 Eliasberg 390

Ascher 107, 188.

Aszalos 387.

B.

Bachstez 86. Bar 103, 308, 309, 328. Baron 99.

Bartels 325, 327, 329. Bartok 199. Beaucamp 290. Beck O.,, 100. Beckershaus 264.

Bergemeister 92, 93. Bergler 100. Beselin 199. Bieling 328, 329. Bielschowsky 112,

118, 120, 123, 197. Bistis 285. Blank 378. Blatt 374, 378· Bliedung 393. Bloch 118, 181. Böhm

77. Braunstein 394. Bruckner 117. Bryn 188, 374. Bus ace a 374.

Caspar 200, 305. Chronis 189. Comberg no, 124, 192,

366. Cords 378. v. Csapody 202. Czellitzer 127.

D.

Dalén 109. David 385.

Elschnig 186, 366, 375,

$39 \mathrm{i}$. 
Engelbrecht 90. j Engelking 367. Enslin 124. Esser 81, 195.

F.

Fazakas 395. Feigenbaum 97, 303. Filatow 388. Fischer, E., 195. Flaschenträger 308. Fleischer 399. Flieringa 379. Fliri 365.

Franceschetti 17. Franke, E., 181. Friede 388. Fuchs, A., 213. Fuchs, E., 102.

G.

Gifford 75. Golowin 394. Greeff 125. Grimminger 74, 85, 92. Groenouw 118. Grüter 364.

Guillery 83, 365. Gutzeit 178, 374.

$\mathrm{H}$.

Hagen 130. H alász 202. Halbertsma 91, 205. Hamann 125. Handmann 101. Hanke 383. Heine 82, 90, 194, 333. Henker 108, 116.

genarbeiten.

Hensen 98, 313, 384,

387. Hentschel 98. Herrenschwand 102. Heßberg 309, 326, 327,

329, 382, 396. Heuser 327. Hillemanns 387. v. Hippel 88, 98, 208. van der Hoeve no,

III.

Hoffmann, W. 85, 103 Hollos 201 Ho ray 204 v. Horváth 184 Huber 383 I. Igersheimer 94, 95.

v. Imre jun. 202. Isakowitz 124, 125,

176.

J. Jablonski 384. Jacoby 395. Jaensch 121, 122, 197,

386. Jäger 84, 307. Jendralski 117. Jeß 77, 88, 102, 310. Jeßner 119. John 401. Judin 201, 375.

Jung 182. J u n g m a n 11 118. Junius 337.

$\mathrm{K}$.

Kaczo ..71. Kägi 80

89

373. Karbe 89. Karpow 106. Kassner 312

326. Kast 357. Kaufmann 134. Kestenbaum 112. Kiel 208. 